\title{
Responses of Japanese Soybeans to Hypoxic Condition at Rhizosphere Were Different Depending upon Cultivars and Ambient Temperatures
}

\author{
Yutaka Jitsuyama \\ Research Faculty of Agriculture, Hokkaido University, Sapporo, Japan. \\ Email: y-jitsu@res.agr.hokudai.ac.jp \\ Received April 11 ${ }^{\text {th }}, 2013$; revised May $12^{\text {th }}, 2013$; accepted June $1^{\text {st }}, 2013$ \\ Copyright (C) 2013 Yutaka Jitsuyama. This is an open access article distributed under the Creative Commons Attribution License, \\ which permits unrestricted use, distribution, and reproduction in any medium, provided the original work is properly cited.
}

\begin{abstract}
To investigate the soybean (Glycine max Merr.) wet endurance, and the affect of the maturity and the ambient temperature to the response, the plantlet in growth stage ranged from R1 to R2 of 8 Japanese soybean cultivars which characterized as various wet endurance in a past report were cultured under hypoxic-hydroponic condition for a month. Two experiments at different periods differed significantly in temperature of air and hydroponic solution, but the oxygenic condition were similar each experiments, as aimed for. And also, control and hypoxia treatments significantly differed in dissolved oxygenic concentration in each experiments. The hypoxic condition at higher temperature induced the reduction of survival of plantlet up to 70\%. At higher temperature, the survival rate of late maturity types "Yuzuru" and "Shirotsurunoko" were decreased significantly in hypoxic condition than in control. Similarly, the shoot dry matters of them were also decreased significantly in hypoxia at higher temperature. The tendency of the decreasing in hypoxia was remarkable in the pod dry matter, and the symptoms were shown also at the lower temperature. From these results, soybean's hypoxic tolerant may be reflected with the wet endurance, the process of hypoxic damages can be divided to sudden death symptom and biomass decreasing, and the mechanisms of hypoxic tolerance might be affected strongly by ambient temperature, and absolutely controlled with the genetic background.
\end{abstract}

Keywords: Ambient Temperature; Converted Field from Paddy; Glycine max Merr.; Hydroponic Culture; Hypoxia; Low Oxygen; Wet Endurance

\section{Introduction}

Heavy rainfall or prolonged rainy periods are sometimes caused the hindering agricultural production. Waterlogging in agricultural field is actually serious problem limiting the crop production in such as eastern Asia or southeastern USA, especially in places with poor drainage and high water tables. The similar cases have happened in Japan. In Japan since 1978, crop production such as soybean, wheat, buckwheat, have been produced at upland field where converted from paddy field, with necessary of adjustment the excess rice production. For soybean production in Japan, $85 \%$ of the cultivation has been conducted at "the converted field" [1]. Generally, high yield could be anticipated if the filed crop was applied to converted field, because of more soil fertility than normal field. However, since the paddy field before conversion normally has a function to keep wet condition for rice production, the converted field is easy to have excess moisture with its high water table and the fine soil particles, clay and silt. Therefore, the production of upland field crop using the converted field sometimes becomes to be unstable just after rainfall. Some technical modifications such as the shift of the productive duration with various maturities and the high ridging cultivation were applied for the avoidance of the waterlogging injury, also the genetic improvement for waterlogging tolerance has been considered [2]. Recently, a new subirrigation and drainage system (FOEAS: Farm-Oriented Enhancing Aquatic System) for controlling water table in converted field has been developed, and was used to avoid wet damage [3]. The advantages of theses modifications were reported a lot, however, the examples of crop waterlogging injury produced in converted field also has been reported every year, it does not lead to the solution to problem.

In case of soybean cultivation at converted field, the 
excess moisture affects negatively to the germination and the growth. One of the waterlogging injury is appeared at germination stage, and the injury have been understood as mainly caused of the collapse of seed structure induced by the rapid water uptake [4,5]. Another waterlogging injury happened in plantlet after germination, it developed with the different mechanism that should be clearly discriminated from seed injury [6,7]. Considering the waterlogging injury in soybean plantlet, many previous studies have demonstrated that the injury harmed physiological functions such as photosynthetic activity, $\mathrm{N}$ fixation and mineral absorption [8-13]. On the other hand, it is reported some certain cases that the soybean plantlet encountered waterlogging situation exhibits adaptive responses by forming adventitious roots [12] or secondary aerenchyma [14]. Thus, many information about wet endurance of soybean has reported, however, the detail of mechanism originating waterlogging injury has not been identified in present.

Except for a few microscopic organisms species, all living creatures, of course including plant root cells, need to uptake oxygen and release carbon dioxide for the respiration. Therefore, the plant growth is severely affected by the gases-ratio condition at the underground, i.e. oxygen: $0 \%-21 \%$, carbon dioxide: $0.03 \%-21 \%$ [15]. The origin of plant watterlogging injury has been understood as their condition, "low oxygen" [16] or "high carbon dioxide" [17-19].

In past researches, the information about the differences in wet endurance among various soybean cultivars has been limited, because of the poor reappearance the expression of waterlogging injury. Many researchers investigated the responsiveness against low oxygenic condition for one cultivar, and the phenologic affects by wet converted field for plural cultivars. Although the comparative physiologic approach is needed for the advance of soybean wet endurance study, the comparative research that many cultivars tested at extremely different gas environment at rhizosphere almost did not be reported. On this study, we firstly simplified the rhizosphere condition using the hydroponic cultured system, so as to remove the buffering effect of the soils and the microorganisms and the affect with nodules. Secondly, we removed oxygen from the hydroponic-solution as possible as lesser level of the concentration. Thirdly, we constructed the experimental equipments like as can be tested with many plantlets of various cultivars at once.

Using improved survey method described above, we investigated the tolerance for low oxygenic condition at rhizosphere (hypoxic tolerance) with 8 soybean cultivars whose field wet endurance are quite unique that Matsukawa et al. picked up in a past research [20]. The main purpose of this study is, thus, check the relationship between the soybean wet endurance in a past research and the hypoxic tolerance in this study. Additionally, we also referred the hypoxic responsiveness expressed in particular cultivars, the relationship between hypoxic responsiveness and ambient temperature, and the relationship between shoot dry matter and pod dry matter.

\section{Materials and Methods}

\subsection{Plant Materials and Experimental Environment}

Soybean (Glycine max Merr.) was used as the experimental material in this study. As see Table 1, 8 cultivars of soybean produced throughout Hokkaido, northern Japan were selected from large variety of maturity. All of them had been researched about the wet endurance at Hokkaido Central Agricultural Experiment Stations [20].

The study was done at the Experimental Farm of the Field Science Center for Northern Biosphere, Hokkaido University, Sapporo, Japan $\left(43^{\circ} \mathrm{N}, 141^{\circ} \mathrm{E}\right)$ in 2007 . Seeds were sown in Vermiculite, and germinated in a incubator at $25^{\circ} \mathrm{C}$ in dark. The soybean seedlings grew to VC growth stage (hypocotyls straightens, and cotyledons unfold) over a period of 1 week, then transferred to hydroponic culture system at greenhouse as a pre-treatment (acclimation to normal hydroponic culture). Soybean seedlings were set the root into $40 \mathrm{~L}$ of hydroponic solution (500

Table 1. Maturity, origin and wet endurance of 8 soybean cultivars used for this study.

\begin{tabular}{|c|c|c|c|c|}
\hline Cultivar $^{1)}$ & Abbreviation & Maturity & Origin & Wet endurance ${ }^{2)}$ \\
\hline Kitakomachi & Kit & Early & Toiku $129 \times$ Toyosuzu & Sensitive \\
\hline Suzuhime & Suz & Medium-early & P184751 × Koganejiro & Moderately tolerant \\
\hline Koganejiro & Kog & Late-early & Zi-hua 4 (from China) $\times$ Tokachinagaha & Tolerant \\
\hline Oshimashirome & Osh & Late-early & Tokachinagaha $\times$ Huang bao zhu & Moderately tolerant \\
\hline Chuseihikariguro & Chu & Late-early & Local soybean variety at Central Hokkaido & Moderately tolerant \\
\hline Yuzuru & Yuz & Late-early & Pure line selection from Tsurunoko & Sensitive \\
\hline Shirotsurunoko & Shi & Extremely_late & Pure line selection from Tsurunoko & Sensitive \\
\hline
\end{tabular}

${ }^{1)}$ These cultivars arrange in according to maturity; ${ }^{2)}$ The wet endurance in converted field evaluated at Agricultural Experiment Station in Hokkaido (Matsukawa et al. 1983). 
times dilution of Hyponica solution, KYOWA Co., Ltd., Osaka, Japan) with stem-holding by the styrene-float (35 $\mathrm{cm} \times 25 \mathrm{~cm}$, thickness: $1 \mathrm{~cm})$ in the container $(50 \mathrm{~cm} \times$ $40 \mathrm{~cm}$, depth: $20 \mathrm{~cm}$ ), and grew to V3 - V4 growth stage with several trifoliate leaves for 1 - 2 weeks (Figure 1(a)). The container has the aeration for all time, and the water surface was completely covered with aluminum sheet for inhibiting growth of algae in the container. In the process of acclimation to hydoroponic culture, these soybean plantlets grow normally with healthy-white roots (Figure 1(b)). A styrene-float had 70 wells, and 50 plantlets were arranged in these wells randomly by a plantlet per a well. A lower part of the stem was supported with plastic-tip of micropipette, and the tip fixed plantlet with styrene-float. For assay of plantlet's response for ambient temperature, the culture was done at two times, at August for higher ambient temperature (High temp.) and October for lower ambient temperature (Low temp.) in 2007. The light conditions of two different periods were adjusted to 16-hour photoperiods (200 $1000 \mu \mathrm{mol} \cdot \mathrm{m}^{-2} \cdot \mathrm{s}^{-1}$ ) using a supplementary artificial light for all equipments.

\subsection{Hydroponic Culture System Able to Be Loaded with Hypoxia}

As equipment for the evaluation of low-oxygen tolerance, simple environment that imposes to the plantlets in the stressed treatment the only low oxygen concentration, was aimed. For the purpose, the hydroponic cultural system was finally selected, it can remove the buffering effect of the soil, and the differences in the response to the hypoxia can be outstood. After the pre-treatment, the soybean plantlets were transferred to another hydroponic culture system $(45 \mathrm{~cm} \times 35 \mathrm{~cm}$, depth: $20 \mathrm{~cm})$ for the treatment of two oxygen conditions, control (Cont.) or hypoxia (Hypoxia) (Figure 1(c)). These plantlets were hydroponic cultured in the system for a month approximately, and grew to R1 - R2 growth stage. The hydroponic culture systems were basically composed of boxes with triple-layer structure (Figure 1(d)), the seedlings floated on $20 \mathrm{~L}$ of hydroponic solution that was composed as same of one in pre-treatment, in the most inside box, and the root of seedlings was grown in this space. In the most outer layer, $4 \mathrm{~L}$ of $10 \%(\mathrm{w} / \mathrm{w})$ sodium sulfite solution was installed as an oxygen absorber (deoxidant), linked with the fertilizer solution through the air in the middle-layer (Hypoxia). The sodium sulfite solution was completely isolated from the external environment with the masking of lid. The system for control treatment has tap water in the most outer space (Cont.), instead of the deoxidant. And also the difference of the system between control and hypoxia treatment is the aeration with airpump at rate of $10 \mathrm{~L} \cdot \mathrm{min}^{-1}$. Both treatments were incorporated the circulation equipment for the equilibrium fertilizer concentration throughout the system, on the
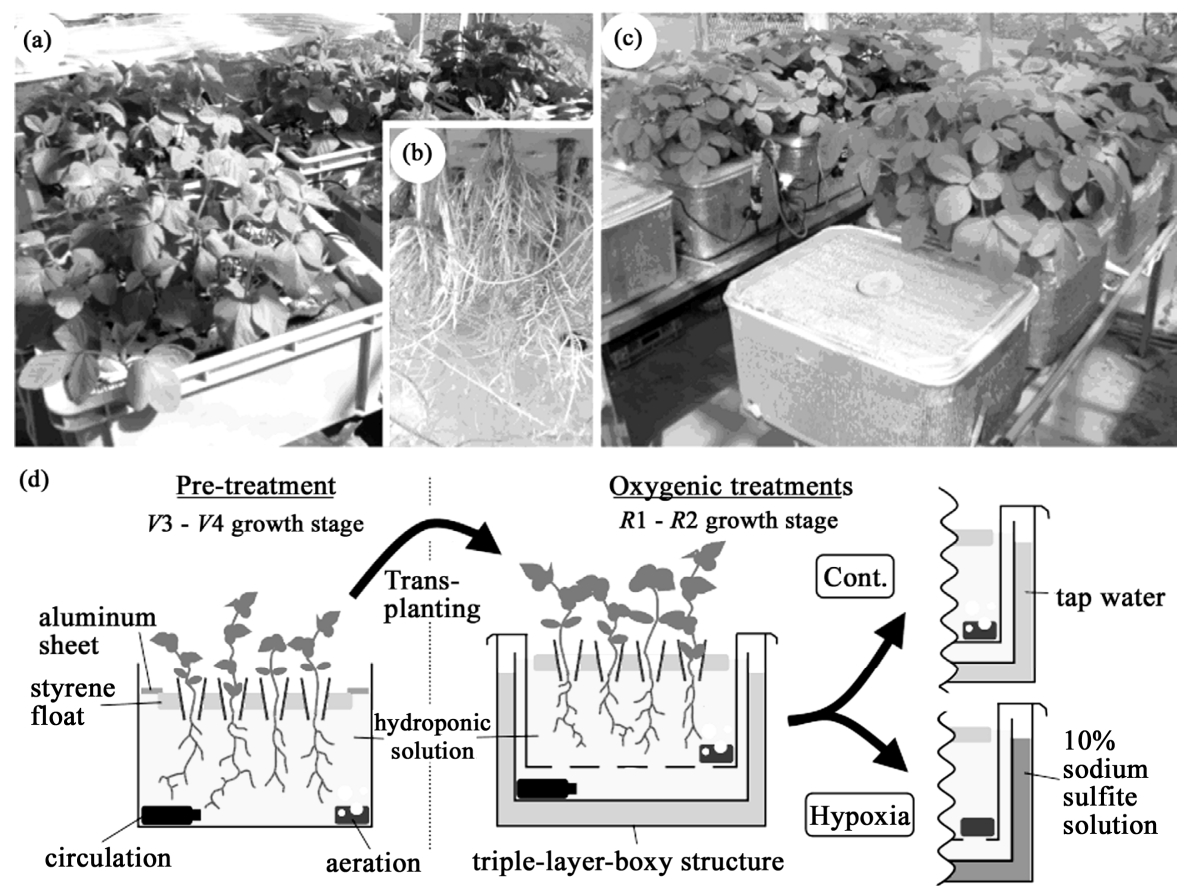

Figure 1. Photographs of soybean plantlets (a) and the roots (b) in pre-hydroponic-culture, and in oxygenic treatments of hydroponic culture (c), and a diagram of this system (d). This system for hypoxia were composed of triple-layer structure, the hydroponic solution link with the surface of liquid at the most outside-layer. In the layer, tap water for control and $10 \%$ (w/w) sodium sulfite for hypoxia were installed each. The system for control has continuous air and, for hypoxia without air. 
Table 2. Temperature, oxygen concentration and $\mathrm{pH}$ of hydroponic culture solution for high and low ambient temperatures.

\begin{tabular}{|c|c|c|c|c|c|c|c|c|c|c|c|c|c|c|c|c|c|}
\hline \multirow{2}{*}{ Ambient temperature } & \multicolumn{6}{|c|}{ Monitored temperature $\left({ }^{\circ} \mathrm{C}\right)$} & \multicolumn{6}{|c|}{ Oxygen concentration $\left(\mathrm{mg} \cdot \mathrm{L}^{-1}\right)$} & \multicolumn{5}{|c|}{$\mathrm{pH}$} \\
\hline & \multicolumn{3}{|c|}{ Air } & \multicolumn{3}{|c|}{ Solution } & \multicolumn{3}{|c|}{ Cont. } & \multicolumn{3}{|c|}{ Нypoxia } & \multicolumn{2}{|r|}{ Cont. } & \multicolumn{3}{|c|}{ Hypoxia } \\
\hline High & 26.7 & $(1.4)^{1)}$ & $* * 2)$ & 25.3 & $(1.0)$ & $* * *$ & 5.7 & $(0.3)$ & & 2.7 & $(0.3)$ & & 7.6 & $(0.1)$ & & 7.4 & $(0.1)$ \\
\hline Low & 18.4 & (2.8) & & 15.3 & (1.3) & & 6.5 & $(0.7)$ & 115 & 2.9 & $(0.5)$ & 115 & 7.9 & $(0.0)$ & ns & 7.6 & $(0.1)$ \\
\hline Oxygen treatment effect & & & & & & & & & & & & & & & *** & & \\
\hline
\end{tabular}

${ }^{1)}$ Values denote standard error; ${ }^{2 * *}$ and ${ }^{* * *}$ represent significant at the 0.01 and 0.001 probability level respectively, ns means no significance with Student-t test between High and Low ambient temperature $(\mathrm{n}=5-24){ }^{3{ }^{* * * *}}$ in the row of oxygen treatment effect represent significant at the 0.001 probability level with Student t-test between control and low oxygen treatment $(n=10-48)$.

bottom of the middle layer. The hydroponic system for a treatment was composed of three boxes with linking each other by polyvinylchloride pipe for the circulation of fertilizer solution. The solution was supplemented $5 \mathrm{~L}$ newly every week. The composition of the hydroponic solution did not change before and after the experiment, was checked with an X-ray Fluorescence Element Analyzer (JSX-3400RII, JEOL Ltd. Tokyo, Japan).

\subsection{Measurements}

The oxygen concentration and $\mathrm{pH}$ of hydroponic solution was intermittently measured with Dissolved Oxygen Meter (DO-5509, Lutron Electronics, Inc., USA) and pH meter (B-211, Horiba ltd., Tokyo, Japan), respectively in same time. The ambient temperature and temperature of hydroponic solution were also measured by thermometer at that time. Every 3 days for the treatment duration, dead plantlets were checked through whole of the system, and rid them out. In the case of study, the plantlet which be suffered the fatal injury was suddenly wilted with keeping the greenish color. Then the shoot and the pod were separately dried at $80^{\circ} \mathrm{C}$ for 3 days more, measured the weight of shoot and pod dry matter.

\subsection{Statistics Analysis}

In this study, the data resulted from a hydroponic box was defined a replication. Two different oxygenic treatments has 3 replications each, the completely randomized block with 3 replications was employed as statistics analysis of this study. For a replication, 5 - 15 plantlets per a cultivar were used, therefore 300 more plantlets were tested in a whole experimental system at once. The statistics analysis including ANOVA and Pearson correlation coefficient were carried out using an SPSS 20.0 Macintosh version statistical package (IBM ${ }^{\circledR}$ SPSS $^{\circledR}$ Statistics, Chicago, USA). Pearson correlation was used to study concordance between shoot and pod dry matter.

\section{Results}

\subsection{Experimental Environment}

The gap of environmental conditions between two dif- ferent experiments mainly could be mentioned to temperature in greenhouse that was significantly different between high and low ambient temperatures with gaps of $10^{\circ} \mathrm{C}$ approximately (Table 2). Dissolved oxygen concentration and $\mathrm{pH}$ of hydroponic culture solution were not significant between high and low ambient temperatures. The significant difference of dissolved oxygen concentration between control (Cont.) and hypoxia (Hypoxia) was made completely, $\mathrm{pH}$ of solution in hypoxia was also lower than in control constantly (Table 2). With varied temperature conditions, the ideal of hydroponic cultured condition which only the rhizosphere has been in low oxygen concentration were realized.

\subsection{Analysis of Variance for All Results}

The averaged values for all results and analysis of variance were shown in Table 3. The significant differences among cultivar could be shown in shoot dry matter (at 0.001 level), they had trend to be smaller in Suz and Kog, larger in Osh and Chu. The significant differences among treatment could shown in survival (at 0.001 level), shoot dry matter (at 0.05 level) and pod dry matter (at 0.001 level). In that case with comparing Cont. and Hypoxia in same temperature-experiment, the significant differences could be shown in survival and pod dry matter in High temp., the value in Cont. were larger than in Hypoxia in both case. Additionally, the affects of replication in any measurements were not significant. The interaction between cultivar and treatment was shown in only shoot dry matter (at 0.001 level), the possibility which the response for treatment were different among cultivar was shown.

\subsection{Changes in Survival of Soybean Plantlets Due to Hypoxia and Ambient Temperature}

The changes in survival of soybean plantlet in timecourse of hydroponic duration was shown in Figure 2. The ambient temperature and oxygenic situation had also the drastic affect to the survival changes in time-course. The survival of plantlet linearly decreased on the timecourse of the hydroponic culture in all experiments, however $70 \%$ more of plantlets survived throughout the 

and Ambient Temperatures

Table 3. Averaged values for survival, shoot dry matter and pod dry matter, and result of ANOVA test for independent variables including cultivar $(C)$, treatment $(T)$ and the interaction $(C \times T)$.

\begin{tabular}{|c|c|c|c|c|c|c|c|c|c|c|}
\hline \multirow{2}{*}{\multicolumn{2}{|c|}{ Cultivar }} & \multicolumn{9}{|c|}{ Measurements } \\
\hline & & \multicolumn{3}{|c|}{ Survival (\%) ${ }^{1)}$} & \multicolumn{3}{|c|}{ Shoot DM (gDW) } & \multicolumn{3}{|c|}{ Pod DM (gDW) } \\
\hline \multicolumn{2}{|c|}{ Kit } & 91.0 & $(5.0)^{2)}$ & & 2.69 & $(0.18)$ & $a b^{3)}$ & 0.155 & $(0.041)$ & \\
\hline \multicolumn{2}{|c|}{ Suz } & 79.0 & (8.4) & & 1.14 & $(0.16)$ & $\mathrm{c}$ & 0.177 & $(0.080)$ & \\
\hline \multicolumn{2}{|c|}{ Toy } & 87.5 & (5.5) & & 3.40 & $(0.27)$ & $\mathrm{ab}$ & 0.346 & $(0.123)$ & \\
\hline \multicolumn{2}{|c|}{ Kog } & 94.4 & (3.7) & & 2.23 & $(0.23)$ & bc & 0.141 & $(0.059)$ & \\
\hline \multicolumn{2}{|c|}{ Osh } & 88.5 & (6.6) & & 3.99 & $(0.47)$ & $\mathrm{a}$ & 0.228 & $(0.051)$ & \\
\hline \multicolumn{2}{|c|}{ Chu } & 86.5 & $(5.2)$ & & 3.79 & $(0.36)$ & $\mathrm{a}$ & 0.256 & $(0.089)$ & \\
\hline \multicolumn{2}{|c|}{ Yuz } & 83.3 & (7.7) & & 3.69 & $(0.33)$ & $\mathrm{ab}$ & 0.231 & $(0.074)$ & \\
\hline \multicolumn{2}{|c|}{ Shi } & 88.9 & (6.3) & & 3.61 & $(0.49)$ & $\mathrm{ab}$ & 0.190 & $(0.094)$ & \\
\hline \multicolumn{11}{|c|}{ Treatment } \\
\hline Temperature & Oxygen & & & & & & & & & \\
\hline \multirow{2}{*}{ High temp. } & Cont. & 96.5 & (1.9) & $\mathrm{a}$ & 3.3 & $(0.32)$ & $a b$ & 0.568 & $(0.065)$ & $\mathrm{a}$ \\
\hline & Нypoxia & 74.0 & (4.9) & $\mathrm{b}$ & 2.3 & $(0.20)$ & $\mathrm{b}$ & 0.146 & $(0.028)$ & $\mathrm{b}$ \\
\hline \multirow{2}{*}{ Low temp. } & Cont. & 95.1 & (2.7) & a & 3.3 & $(0.27)$ & $a b$ & 0.093 & $(0.018)$ & b \\
\hline & Hypoxia & 83.9 & (5.2) & $\mathrm{ab}$ & 3.4 & $(0.32)$ & $\mathrm{a}$ & 0.054 & $(0.018)$ & $\mathrm{b}$ \\
\hline ANOVA & $\mathrm{df}$ & MS & & $\mathrm{P}$ & MS & & $\mathrm{P}$ & MS & & $\mathrm{P}$ \\
\hline \multirow[t]{2}{*}{ Cultivar (C) } & 7 & 264.337 & & $\mathrm{~ns}^{4)}$ & 11.519 & & $* * *$ & 0.187 & & ns \\
\hline & 3 & 2685.352 & & $* * *$ & 7.091 & & $* * *$ & 2.778 & & $* * *$ \\
\hline Replication & 2 & 121.387 & & ns & 0.664 & & ns & 0.227 & & ns \\
\hline $\mathrm{C} \times \mathrm{T}$ & 21 & 474.605 & & ns & 2.314 & & $* * *$ & 0.215 & & ns \\
\hline
\end{tabular}

${ }^{1)}$ Survival of soybean plantlet was checked at $28^{\text {th }}$ day of hydroponic culture; ${ }^{2)}$ Values denote standard error; ${ }^{3)}$ Values with different letters are significantly different at the 0.05 probability level by Tukey-Kramer's test; ${ }^{4)}{ }_{* * *}^{* *}$ represent significant at the 0.001 probability level, ns means no significance with ANOVA test.

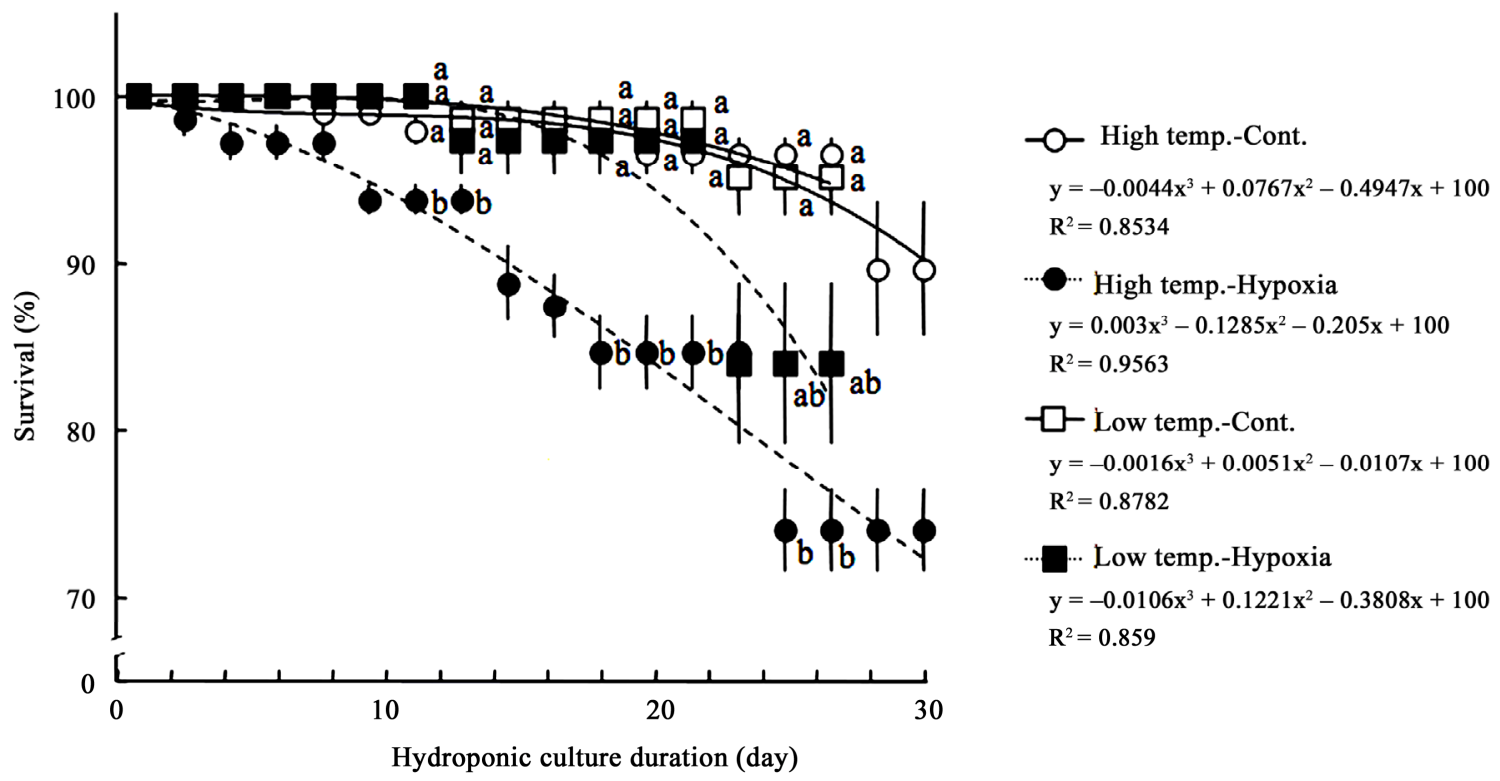

Figure 2. Changes in survival of soybean plantlet due to hydroponic culture duration. Circle and square symbols show the values in high $(\circ, \bullet)$ and low $(\square, \square)$ ambient temperature, respectively. Open and Close symbols show the values of Cont. $(\circ, \square)$ and Hypoxia $(\bullet, \bullet)$, respectively. Data represent means from 3 preparations, and bars show standard errors. The regression lines are for the means of each treatment, were shown the formula below each symbol. Values with different letters are significantly different at the $\mathbf{0 . 0 5}$ probability level by Tukey-Kramer's test on same day. 
hydroponic culture actually. As a tendency, the decrease of survival was late in Cont., and was fast in Hypoxia. The other trend was that the survival decreased faster in High temp. than in Low temp. Particularly, the survival in Low temp.-Hypoxia decreased significantly in compared with survivals in other treatments after $10^{\text {th }}$ day of hydroponic culture intermittently.

The differences in survival among 8 cultivars were not significant in both temperatures and both oxygenic treatments (all these four provability $>0.1$, Table 3 ), however, the survival in the end of treatment of Hypoxia were significantly lower than that of Cont. in Yuz (at 0.05 level), Shi (at 0.05 level) and Suz (at 0.1 level) in High temp (Figure 3). However, the other cultivars including in Low temp., also showed trend of the survival decrease due to the hypoxia treatment, they were not significant. In High temp., only one cultivar, Kog kept the $100 \%$ of survival even if in Hypoxia.

\subsection{Shoot and Pod Dry Matter after a Monthly Hydroponic Culture}

The survived soybean plantlets after hydroponic culture were used for the measurement of biomass, the differences in shoot dry matter among cultivars due to hydroponic culture with hypoxia was shown in Figure 4. The variation in shoot dry matter were ranged between 0.87 and $5.79 \mathrm{~g}$ DW broadly, the average was $3.07 \mathrm{~g}$ DW. The shoot dry matter in Low temp. Hypoxia was larger significantly than in High temp. Hypoxia (Table 3), and the trend was also shown in Figure 4. Some cultivars, Chu (at 0.05 level) and Shi (at 0.1 level) in High temp., Kit (at 0.05 level) and Kog (at 0.1 level) in Low temp., showed significant decrease shoot dry matter due to Hypoxia. These cultivar-specific responses to the treatments affected the significant interaction between cultivars and treatments were detected in shoot dry matter (at 0.001 level, Table 3).

Many plantlets accomplished to the reproductive growth stage in this hydroponic culture system for one month, and had pods but some lean. The data of pod dry matter was also shown in Figure 5, to compare them among cultivars and treatments. The variation in pod dry matter of soybean plantlets were ranged from $4.4 \mathrm{mg}$ DW to 0.997 g DW broadly as like shoot dry matter. Generally, the pod dry matter in High temp. had trend to be larger than in Low temp., and the pod dry matter in Cont. had trend to be larger than in Hypoxia. Then, especially the pod dry matter in High temp.-Cont. showed significantly larger value than in other treatments (at 0.05 level, Table 3). Many cultivars showed the decrease pod dry matter due to Hypoxia, the significant decrease detected Osh (at 0.01 level), Chu (at 0.05 level) and Shi (at 0.05 level) in High temp., Kit (at 0.01 level), Toy (at 0.05 level) and Chu (at 0.05 level) in Low temp., respectively.

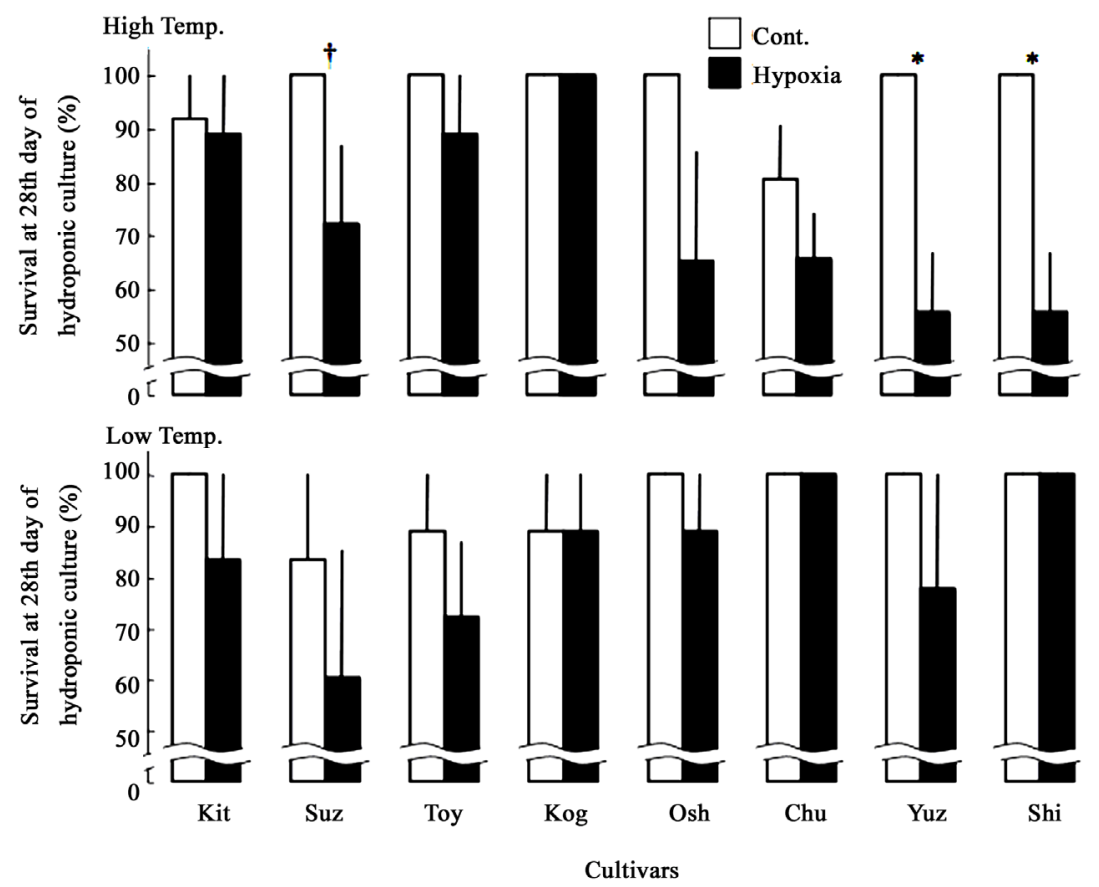

Figure 3. Differences in survival of plantlet at $28^{\text {th }}$ day of hydroponic culture among cultivars due to low oxygen concentration. $\square$ : Cont., $\square$ : Hypoxia. Data represent means from 3 preparations, and bars show standard errors. $\dagger$ and $*$ represent significant at the 0.1 and 0.05 probability level, respectively, with t-test between control and hypoxia. The values are arranged according to the maturity shown in Table 1. 


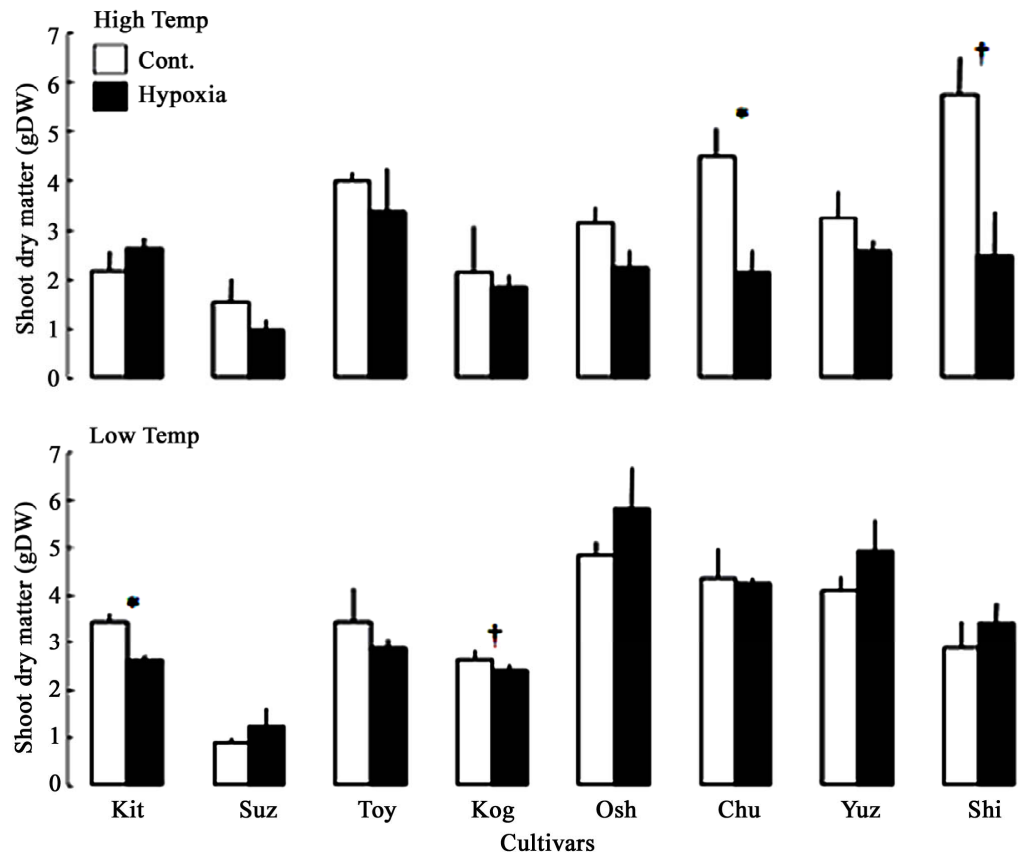

Figure 4. Differences in shoot dry matter among cultivars due to hydoroponic culture with low oxygen concentration. $\square$ : Cont., $\square$ : Hypoxia. Data represent means from 3 preparations, and bars show standard errors. $\dagger$ and * represent significant at the 0.1 and 0.05 probability level, respectively, with t-test between control and hypoxia. The values are arranged according to the maturity.

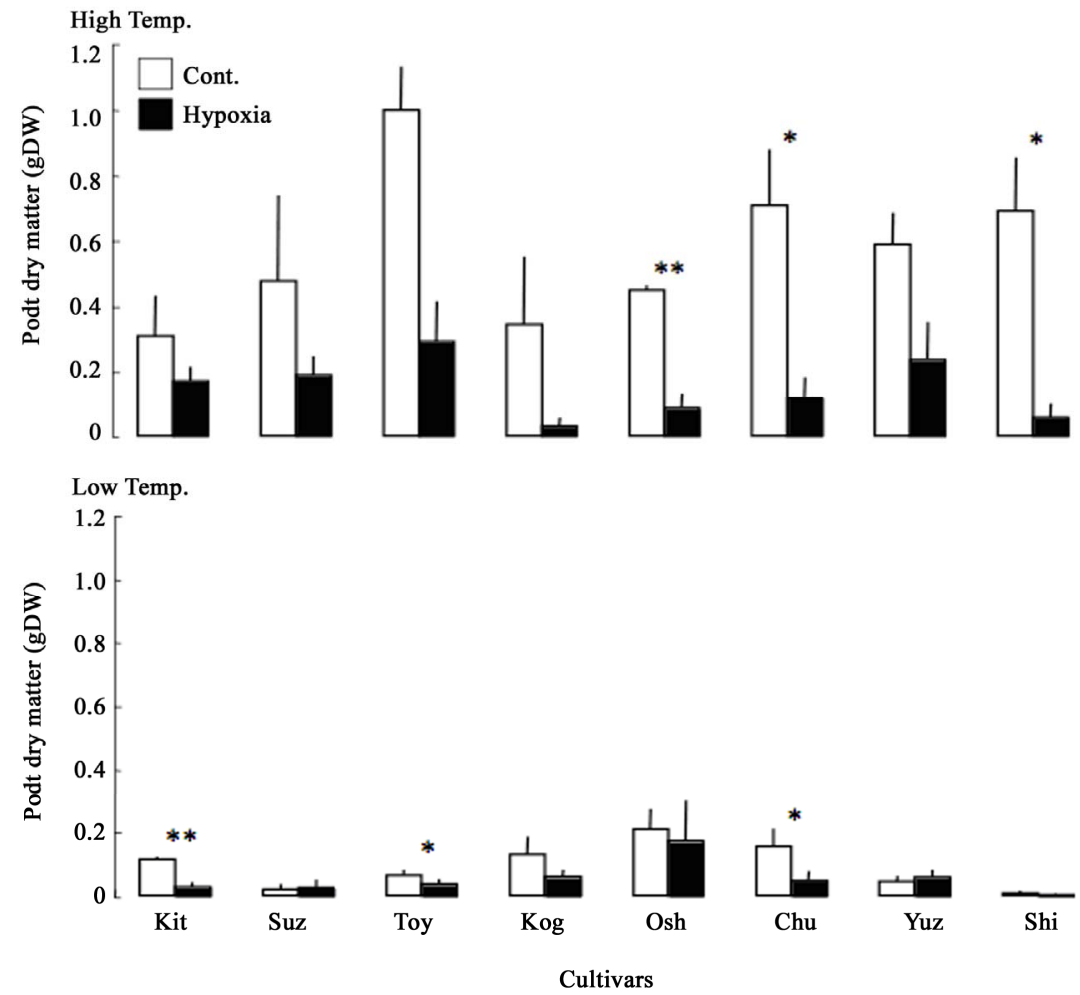

Figure 5. Differences in pod dry matter among cultivars due to hydoroponic culture with low oxygen concentration. $\square$ : Cont. 口: Hypoxia. Data represent means from 3 preparations, and bars show standard errors. * and ** represent significant at the 0.05 and 0.01 probability level, respectively, with t-test between control and hypoxia. The arrangements of values are according to the maturity. 
We also defined the magnitude of decrease dry matter due to hypoxia situation using the value of Cont./Hypoxia (Table 4). The magnitude decrease dry matter due to hypoxia was significantly larger in pod than in shoot, nevertheless in High or Low temp. situation. The difference in the magnitude of decrease in the dry matter due to hypoxia was bigger in High temp. (at 0.01 level) than in Low temp. (at 0.05 level).

\subsection{Relationships between Shoot Dry Matter and Pod Dry Matter}

As shown in Figure 6, the relationship between shoot dry weight and pod dry weight was significant in High temp. $\left(\mathrm{R}^{2}=0.4825^{* *}, \mathrm{n}=16\right)$ and in Low temp. $\left(\mathrm{R}^{2}=\right.$ $0.3533^{*}, n=16$ ), however, the relationships of them for all data in both temperature treatments was not signifi- cant $\left(\mathrm{R}^{2}=0.0854^{\mathrm{ns}}, \mathrm{n}=32\right)$.

\section{Discussion}

\subsection{Making the Hypoxia at Rhizospere}

In this study, we applied the hydroponic culture system with low oxygenic condition at rhizosphere as possible as simplified, to the evaluation for wet endurance of soybean plantlet. The presence of soil has the various buffering effect at the underground, and the presence of nodule also has large affect to soybean responsiveness against low oxygenic condition via the reductions of the nodulation [21], and of the rate of nitrogen fixation [12]. We removed theses affective factors from the experimental equipment, so that the hypoxic tolerance of soybean plantlet can be detected independently.

Table 4. T-test for comparison between shoot and pod, for the magnitude of decrease due to Hypoxia (Cont./Hypoxia).

\begin{tabular}{cccccc}
\hline Temperature & Types of dry matter & \multicolumn{2}{c}{ Cont./Hypoxia } & df & Probability \\
\hline Total & Shoot & 1.235 & $(0.115)^{1)}$ & 15 & $0.00275^{* * 2)}$ \\
& Pod & 3.875 & $(0.886)$ & & \\
High temp. & Shoot & 1.488 & $(0.181)$ & 7 & $0.00977^{* *}$ \\
& Pod & 5.730 & $(1.485)$ & & \\
Low temp. & Shoot & 0.981 & $(0.073)$ & 7 & $0.01256^{*}$ \\
& Pod & 2.021 & $(0.420)$ & & \\
\hline
\end{tabular}

${ }^{1)}$ Values denote standard error; ${ }^{2)}{ }^{*}$ and ${ }^{* *}$ represent significant at the 0.05 and 0.01 probability level respectively, with t-test between shoot and pod dry matter $(n=3)$.

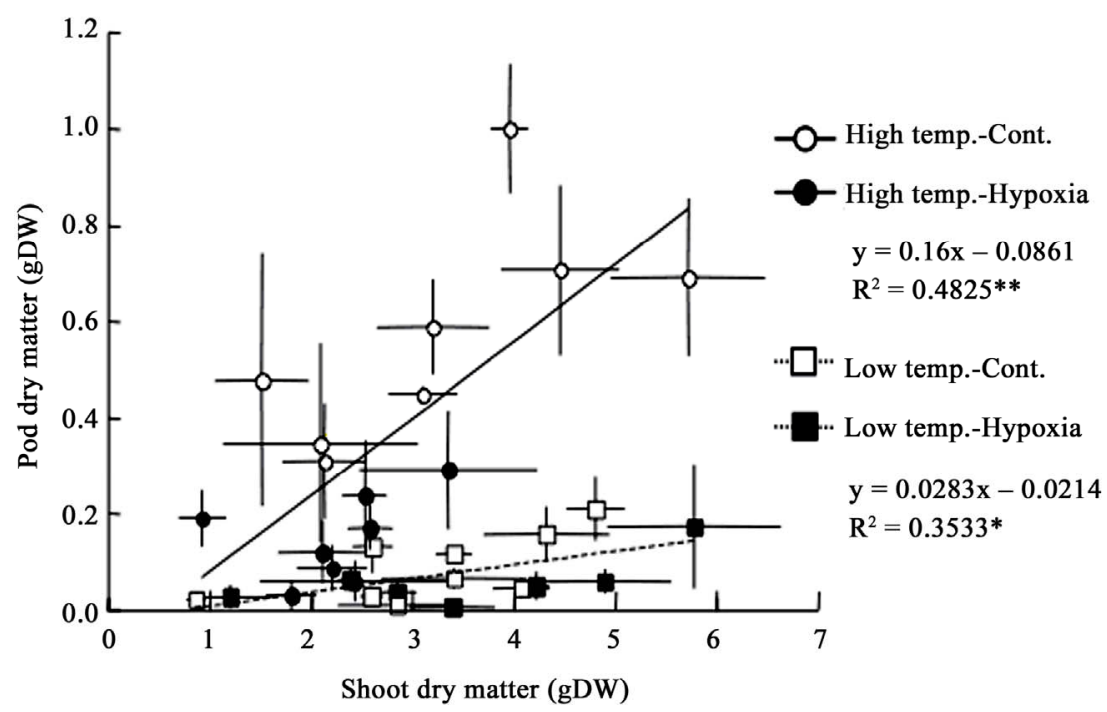

Figure 6. Changes in relationship between shoot dry matter and pod dry matter due to hydroponic culture at different temperature. Circle and square symbols show the values in high $(O, \bigcirc)$ and low $(\square, \square)$ ambient temperature, respectively. Open and Close symbols show the values of Cont. $(O, \square)$ and Hypoxia $(\odot, \square)$, respectively. Data represent means from 3 preparations, and bars show standard errors. The regression lines are for the means of each treatment, were shown the formula below each symbol. * and ** represent significant at the 0.05 and 0.01 probability level, respectively, with Pearson's correlation coefficient. 
A significant difference in oxygenic concentration between Cont. and Hypoxia was constructed as our aim, with two factors of the aeration in Cont. and the incorporation of deoxidant in Hypoxia. We selected the solution of $10 \%(\mathrm{w} / \mathrm{v})$ of sodium sulfite as a deoxidant in this experiment. The solution was used as zero standard solution for the adjustment of dissolved oxygen meter. We confirmed that this solution made low-partial oxygen pressure at sealed space in the experimental equipment according to "Henry's law", and then removed moderately and continuously oxygen from the hydroponic solution. Adding the effect of aeration in Cont. with deoxidant innHypoxia, it was accomplished that the oxygen concentration Hypoxia was always 50\%-lower than that in Cont. (Table 2).

In this study, the $\mathrm{pH}$ of hydroponic solution was measured, because we considered a possibility that the carbon dioxide concentration could reflect to the $\mathrm{pH}$. Some researchers supported that high carbon dioxide concentration was main factor for waterlogging stress rather than low oxygen condition [17-19]. Greenway et al. showed that the differentiations of cultivar's response between treatments were detected when the partial pressure of dissolved carbon dioxide concentration was increased form 5 to $75 \mathrm{kPa}$ at waterlogged field [18]. Boru et al. also concluded that the essence of waterlogging injury of soybean was the carbon dioxide toxicity, using a cultivar "Williams" hydroponic-cultured with carbon dioxide gas [17]. Irving et al. suggested that waterlogging injury was caused the $15 \%$ of increasing in carbon dioxide accompany with $20 \%$ of decreasing in oxygen, in their pot experiment using lucerne [19]. In contrast, Bouma et al. showed no toxic effect of carbon dioxide to root respiration and the elongation using bean plant [16]. In the experimental equipment, actually, the $\mathrm{pH}$ in $\mathrm{Hy}-$ poxia treatment was significantly decreased in compared with Cont., and this may mean that the solution in Hypoxia was acidified by increasing carbon dioxide (Table 2). At last, we could not separate two factors of oxygen and carbon dioxide in this study, however, total gases condition that induces soybean waterlogging injury could equipped completely.

\subsection{Response against Hypoxia—Sudden Death Caused by Hypoxia in Rhizosphere}

On field study in USA, the waterlogging impact on soybean production appeared not only in the biomass decrease but also in the plant survivability [22]. In this study, the survival of soybean plantlet in the hydroponic culture was gradually decreased for a month at rage from $70 \%$ to $95 \%$, and the magnitude of decreasing was larger in hypoxia treatment, and in higher ambient temperature (Figure 2). And the significant decrease in survival due to hypoxia treatment was shown in two cultivars, Yuzuru and Shirotsurunoko tested at higher temperature (Figure 3). The symptom of the dead was suddenly happened with rapid wilting of upper leaves, and then that spread to all leaves and stem of plantlet completely within 3 days 1 week from the begging of the wilting. These results indicate that the sudden death of soybean plantlet due to hypoxia differently happened depending upon the cultivar's variation, and the rapid wilting may be promoted with higher temperature. Matsukawa et al. also reported that waterlogging injury and the death were increased in high water-temperature in waterlogging field, and concluded that the phenomenon originated from the root physiological damage [20]. The transpiration on plant leaf generally increases at higher temperature than that at lower, i.e. increase at range of $4 \%-5 \%{ }^{\circ} \mathrm{C}^{-1}$ in case of soybean [23]. The sudden death of soybean in this study may be explained as the result of 'embolism', if the root water uptake was inhibited nevertheless the transpiration accelerated at the higher ambient temperature. Although the embolism in waterlogging-plant like as rice can be reversible with nighttime-refill water in vessels [24], the embolism is irreversible so that the secretion of water is broken and the water transport is inhibited in general plant [25]. The sudden death injury by wilting soybean plantlet might be happen with the irreversible embolism at high temperature.

For the expression of the embolism in waterlogging situation described above, the interference of root water uptake due to low oxygenic condition is necessary as a premise. The anaerobic response of plants, including soybean also, has been studied focusing on how roots are injured by oxygenic deficiency and how metabolism changes during acclimation to low oxygenic concentration, using the condition of hypoxia or anoxia [26-31]. One early response of plants to anoxia is down regulation of water uptake due to inhibition of the water permeability (hydraulic conductivity) of roots, mediated with water channel proteins (aquaporins) of plasma membrane intrinsic protein [29]. Using Arabidopsis, Colette et al. (2003) reported that the inhibition of water uptake with aquaporins was stimulated sharply by cytosolyc acidosis, which could be a trigger of sudden wilting of plant. In the future, the comparison the water permeability and the expression of aquaporins in root among various cultivars, will be indispensable to clear the differentiation among cultivar's wet endurance.

As the origin of the sudden death of soybean plantlet due to low oxygenic condition, we also considered the possibility of affection by "Phytophthora root and stem rot". At the waterlogging situation, Phytophthora root and stem rot is easy to develop in soybean cultivation [32]. We could not observe the spore or the spawn of $P$. 
sojiaein the sample's tissue, however, it should be sterilized with pesticides before experiment for next time.

\subsection{Other Response against Hypoxia-Biomass Decline Caused by Hypoxia in Rhizosphere}

The decrease of soybean shoot dry matter due to hypoxic condition was significant, in particular cultivars "Chuseihikariguro" and "Shirotsurunoko" at High temp., and "Kitakomachi" at Low temp. (Figure 4). In ANOVA, the significant interaction between cultivars and treatments at shoot dry matter (Table 3), and we interpreted that the interaction originated from the cultivar-specific responses to hypoxia above. The trend of decrease in dry matter was remarkable in pod comparing with in shoot. The decrease of soybean pod dry matter due to hypoxic condition was significant, in particular cultivars "Oshimashirome", "Chuseihikariguro" and "Shirotsurunoko" at High temp., and 'Kitakomachi', "Toyosuzu” and "Chuseihikariguro" at Low temp. (Figure 5). Generally in the pod dry matter, the values at High temp. were larger than at Low temp., and the values at Cont. were larger than at Hypoxia, similarly among many cultivars. These trends might reflect to that the interaction between cultivars and treatments was not significant in ANOVA (Table 3).

As a reason for the biomass reduction due to hypoxic condition, we considered the possibility that the photosynthetic activity was limited by water use deficiency with "partial interruption" in the vessels. In case of the simple water deficiency with drought, soybean plantlet decreased the stomatal conductance, the leaf turgor and then the expansion [33], and decreased severely the biomass production and the yield of crops [34]. On the other hand, Oosterhuis et al. showed that the waterlogging condition induced the reduction of water potential in leaf and decrease in dry matter production, but not affected to the photosynthetic activity or the stomatal conductance in their field experiment with 2 cultivars of soybean [10]. Thus, the reason of plant biomass reduction in case of hypoxia, not in case of simple drought, has not been clarified. Further research is needed to identify the mechanism that hypoxic condition links to biomass reduction of soybean.

The correlation coefficient with the relationship between shoot dry matter and pod dry matter was not significant for a regression line in the all data, but were significant in both each case High and Low temp. (Figure 6). This result indicates the close relationship between shoot biomass and pod biomass, and the relationships were affected by the ambient temperature. As a trend of dot-distributions, data in Hypoxia concentrated in range of low pod dry matter at High temp. comparing with at Low temp. (Figure 6). This trend is also clear at the magnitude of pod dry matter reduction due to hypoxia at
High temp. (Figure 5). From these results, it was cleared that the pod biomass responded sharply to hypoxic condition at higher temperature than at lower. Pod production of soybean subtly respond to the nitrogen supply from fixation with nodulation [12], and the nitrogen played a great role as a supportable nutrients in waterlogging-induced soybean yield losses [13]. Although the hydroponic system in this study had no addition of any nodules, we gave enough amount of nitrogen to cultured plantlet. We can interpret that the differentiation in responsiveness to hypoxia among cultivars present without the affect of nodulation, and the hypoxia condition affect directly to nitrogen uptake through the stem and root.

Additionally, the reduction of inorganic uptake including nitrogen not only induced the reduction of transpiration and photosynthetic ability and also accelerated flower abscission that resulted to yield loss [6]. In this study, we did not count the number of flower, however, the relationship between the reduction of biomass delivered to pod via flower abscission and the pod responsiveness to Hypoxia - High temp., were considered.

\subsection{Differences in Hypoxia Tolerance among Cultivars and the Appropriateness as an Evaluative for Wet Endurance}

The study that focused on the differences of cultivars in waterlogging tolerance is less $[2,20,35]$, the report that discussed about soybean maturity and wet endurance is rare [2]. Mochizuki et al. indicated no relationship between the maturity and the wet endurance in their waterlogging experiment, the wet endurance might be originated from the cultivars which have the traits of less vegetative-biomass decreasing with excess moisture. Matsukawa et al. conducted the wet endurance test using soybeans of 19 cultivars and 1 variety bred in Hokkaido, northern Japan, did not refer the maturity to differences in wet endurance, but showed the genetic background of tolerant cultivars [20]. We picked up 8 cultivars with various maturities from a Matsukawa's report, the survivability and the biomass also had no relationship between the maturity of cultivars and the hypoxic tolerance (Figures 3-5). In the results, the wet endurance or hypoxic tolerance may be independent to the maturity of plantlet.

In viewpoints of the survivability and the biomass of shoot and pod described above, we could attend a cultivar "Shirotsurunoko" that has a unique characteristic, the sensitiveness for hypoxic condition. The cultivar was bred from just one cultivar "Tsurunoko" that has trait of good taste for boiled bean but sensitive to cyst nematode and soybean dwarf virus. Considering that "Yuzuru" was evaluated as a sensitive cultivar against hypoxia in the survivability, and was also bred from "Tsurunoko", the 
relationship between the genetic background of "Tsurunoko" and the sensitiveness against hypoxia was guessed. And we could also attend a cultivar "Koganejiro" with a quite unique characteristic that no affect of hypoxia was shown at high ambient temperature, nevertheless the remained cultivars all-affected. "Koganejiro" was evaluated as a waterlogging tolerant cultivar in compared with other cultivars, when they were suffered by local heavy rainfall at central Hokkaido in 1981 [36]. From theses coincidence with different experimental theories, we guessed that the hypoxic hydroponic culture system may be appropriate as a survey method to find the unique hypoxic tolerant soybean cultivar, and as a technique for the evaluation of soybean wet endurance.

\section{Conclusion}

We indicated the differences in shoot responsiveness to hypoxia condition at rhizosphere among various soybean cultivars with simplified hydroponic culture system. Also the relationship between the hypoxic tolerance in this study and the evaluation of wet endurance in the past research might be reasonable in part of cultivars. In the next step of this study, we will focus measurement targets on more traits including root, with more soybean cultivars who are of unique wet endurance including "Koganejiro" and "Shirotsurunoko". And we will modify the experimental equipment as to make the treatmentduration shortened, with younger plantlet comparing with this trial. For that modification, the effectiveness of oxygenic remove from hydroponic solution will be necessary to widen a difference of oxygen concentration between Cont. and Hypoxia. These investigations may clarify the process of severe soybean waterlogging injury, and these basic information will link to the breeding improvement of soybean production at the converted field.

\section{Acknowledgements}

This study was supported by Grant-in-Aid for Scientific Research from the Japan Society for the Promotion of Science (nr. 22688002). We thank Mr. S. Ichikawa of the Field Science Center for the Northern Biosphere, Hokkaido University, for help with constructing the experimental system.

\section{REFERENCES}

[1] Ministry of Agriculture, Forestry and Fisheries (MAFF), Japan, “Soybean Planting Area in 2012 (Dry Yield)," Statistics of Agriculture, Forestry and Fisheries, Department of Minister's Secretariat Statistics, 2012.

http://www.maff.go.jp/j/tokei/kouhyou/sakumotu/mensek i/pdf/sakutuke_daizu_12.pdf

[2] T. Mochizuki and S. Matsumoto, "Varietal Differences of
Wet Endurance in Autumn Soybean Plants,” Japanese Journal of Crop Science, Vol. 60, No. 3, 1991, pp. 380-384. (In Japanese, with summary in English) doi:10.1626/jcs.60.380

[3] S. Shimada, "Enhancing Soybean Productivity in the Paddy Fields in Japan,” Proceeding of the Congress, International Crop Science Society, 2008.

http://www.intlcss.org/files/congress-proceedings/2008-p apers/cs1-s1/cs1-s1-o6-shinji-shimada.pdf

[4] A. C. Leopold, "Temperature Effects on Soybean Imbibition and Leakage,” Plant Physiology, Vol. 65, No. 6, 1980, pp. 107-109.

[5] X.-H. Tian, T. Nakamura and M. Kokubun, "The Role of Seed Structure and Oxygen Responsiveness in Pre-Germination Flooding Tolerance of Soybean Cultivars," Plant Production Science, Vol. 8, No. 2, 2005, pp. 157165. doi:10.1626/pps.8.157

[6] H. Sugimoto, "Excess Moisture Injury of Soybean Cultivated in a Drained Paddy Field," Memoirs of the College of Agriculture, Ehime University, Vol. 39, No. 1, 1994, pp. 75-134. (In Japanese, with summary in English)

[7] N. Nakayama, S. Shimada, M. Takahashi, Y. -H. Kim and J. Arihara, "Effects of Water-Absorbing Rate of Seed on Flooding Injury in Soybean,” Japanese Journal of Crop Science, Vol. 74, No. 3, 2005, pp. 325-329. (In Japanese, with summary in English) doi:10.1626/jcs.74.325

[8] J. L. Griffin and A. M. Saxton, "Response of Solid-Seeded Soybean to Flood Irrigation. II. Flood Duration,” Agronomy Journal, Vol. 80, No. 6, 1988, pp. 885-888. doi:10.2134/agronj1988.00021962008000060009x

[9] H. D. Scott, J. De Angulo, M. B. Daniels and L. S. Wood, "Flood Duration Effects on Soybean Growth and Yield," Agronomy Journal, Vol. 81, No. 4, 1989, pp. 631-636. doi:10.2134/agronj1989.00021962008100040016x

[10] D. M. Oosterhuis, H. D. Scott, R. E. Hampton and S. D. Wullschleger, "Physiological Responses of Two Soybean (Glycine max (L.) Merr.) Cultivars to Short-Term Flooding,” Environmental and Experimental Botany, Vol. 30, No. 1, 1990, pp. 85-92. doi:10.1016/0098-8472(90)90012-S

[11] F. J. M. Sung, "Waterlogging Effect on Nodule Nitrogenase and Leaf Nitrate Reductase Activities in Soybean," Field Crops Research, Vol. 35, No. 3, 1993, pp. 183-189. doi:10.1016/0378-4290(93)90152-D

[12] M. Bacanamwo and L. C. Purcell, "Soybean Dry Matter and N Accumulation Responses to Flooding Stress, N Sources and Hypoxia," Journal of Experimental Botany, Vol. 50, No. 334, 1999, pp. 689-696. doi:10.1093/jxb/50.334.689

[13] J. E. Board, "Waterlogging Effects on Plant Nutrient Concentrations in Soybean," Journal of Plant Nutrition, Vol. 31, No. 5, 2008, pp. 828-838. doi:10.1080/01904160802043122

[14] S. Shimamura, T. Mochizuki, Y. Nada and M. Fukuyama, "Secondary Aerenchyma Formation and Its Relation to Nitrogen Fixation in Root Nodules of Soybean Plants (Glycine max) Grown under Flooded Conditions,” Plant 
Production Science, Vol. 5, No. 4, 2002, pp. 294-300. doi:10.1626/pps.5.294

[15] J. E. Plaster, "Soil Is a Medium for Plant Growth,” In: J. E. Plaster, Ed., Soil Science \& Management, 2nd Edition, Delmar Publishers Inc., New York, 1992, pp. 6-7.

[16] T. J. Bouma, K. L. Nielsen, D. M. Eissenstat and J. P. Lynch, "Soil $\mathrm{CO}_{2}$ Concentration Does Not Affect Growth or Root Respiration in Bean and Citrus," Plant, Cell and Environment, Vol. 20, No. 12, 1997, pp. 1495-1505. doi:10.1046/j.1365-3040.1997.d01-52.x

[17] G. Boru, T. Vantoai, J. Alves, D. Hua and M. Knee, "Responses of Soybean to Oxygen Deficiency and Elevated Root-Zone Carbon Dioxide Concentration," Annals of Botany, Vol. 91, No. 4, 2003, pp. 447-453. doi:10.1093/aob/mcg040

[18] H. Greenway, W. Armstrong and T. D. Colmer, "Conditions Leading to High $\mathrm{CO}_{2}(>5 \mathrm{kPa})$ in WaterloggedFlooded Soils and Possible Effects on Root Growth and Metabolism,” Annals of Botany, Vol. 98, No. 1, 2006, pp. 9-32. doi:10.1093/aob/mcl076

[19] L. J. Irving, Y. -B. Sheng, D. Woolley and C. Matthew, "Physiological Effects of Waterlogging on Two Lucerne Varieties Grown under Glasshouse Conditions,” Journal of Agronomy and Crop Science, Vol. 193, No. 5, 2007, pp. 345-356. doi:10.1111/j.1439-037X.2007.00277.x

[20] I. Matsukawa, Y. Tanimura, R. Teranishi and H. Banba, "Varietal Difference of Resistance to Excess Wet Injury of Soybean in Dry Field Converted from Paddy Rice Field," Report of Hokkaido Central Agricultural Experiment Station, Vol. 49, No. 1, 1983, pp. 32-40. (In Japanese, with summary in English)

[21] F. R. Minchin and J. S. Pate, "Effects of Water, Aeration, and Salt Regime on Nitrogen Fixation in a Nodulated Legume-Definition of an Optimum Root Environment," Journal of Experimental Botany, Vol. 2626, No. 1, 1975, pp. 60-69. doi:10.1093/jxb/26.1.60

[22] M. Sullivan, T. Van Toai, N. Fausey, J. Beuerlein, R. Parkinson and A. Soboyejo, "Evaluating On-Firm Flooding Impacts on Soybean,” Crop Science, Vol. 41, No. 1, 2001, pp. 93-100. doi:10.2135/cropsci2001.41193x

[23] P. Jones, L. H. Allen and J. W. Jones, "Responses of Soybean Canopy Photosynthesis and Transpiration to WholeDay Temperature Changes in Different $\mathrm{CO}_{2}$ Environments," Agronomy Journal, Vol. 77, No. 2, 1985, pp. 242-249. doi:10.2134/agronj1985.00021962007700020016x

[24] S. Volker, J. S. Sperry and R. Lafitte, "Embolized Conduits of Rice (Oryza sativa, Poaceae) Refill Despite Negative Xylem Pressure," American Journal of Botany, Vol. 92, No. 12, 2005, pp. 1970-1974. doi:10.3732/ajb.92.12.1970

[25] U. G. Hacke, V. Stiller, J. S. Sperry, J. Pittermann and K. A. McCulloh, "Cavitation Fatigue: Embolism and Refilling Cycles Can Weaken the Cavitation Resistance of Xylem,” Plant Physiology, Vol. 125, No. 2, 2001, pp. 779786.

[26] D. A. Russell, D. M. L. Wong and M. M. Sachs, “The
Anaerobic Response of Soybean,” Plant Physiology, Vol. 92, No. 2, 1990, pp. 401-407. doi:10.1104/pp.92.2.401

[27] J. K. M. Roberts, M. A. Hooks, A. P. Miaullis, S. Edwards and C. Webster, "Contribution of Malate and Amino Acid Metabolism to Cytoplasmic pH Regulation in Hypoxic Maize Root Tips Studied Using Nuclear Magnetic Resonance Spectroscopy,” Plant Physiology, Vol. 98, No. 2, 1992, pp. 480-487.

[28] C. D. Malcolm, “Oxygen Deficiency and Root Metabolism: Injury and Acclimation under Hypoxia and Anoxia," Annual Review of Plant Physiology and Plant Molecular Biology, Vol. 48, No. 1, 1997, pp. 223-250. doi:10.1146/annurev.arplant.48.1.223

[29] T. R. Colette, M. Sutka, H. Javot, E. Gout, P. Gerbeau, D. T. Luu, R. Bligny and C. Maurel, "Cytosolic pH Regulates Root Water Transport during Anoxic Stress through Gating of Aquaporins,” Nature, Vol. 425, No. 6956, 2003, pp. 393-397. doi:10.1038/nature01853

[30] W. G. Choi and D. M. Roberts, “Arabidopsis NIP2;1, a Major Intrinsic Protein Transporter of Lactic Acid Induced by Anoxic Stress,” The Journal of Biological Chemistry, Vol. 282, No. 33, 2007, pp. 24209-24218. doi:10.1074/jbc.M700982200

[31] F. Shi, R. Yamamoto, S. Shimamura, S. Hiraga, N. Nakayama, T. Nakamura, K. Yukawa, M. Hachinohe, H. Matsumoto and S. Komatsu, "Cytosolic Ascorbate Peroxidase 2 (cAPX 2) Is Involved in the Soybean Response to Flooding," Phytochemistry, Vol. 69, No. 6, 2008, pp. 12951303. doi:10.1016/j.phytochem.2008.01.007

[32] V. T. Nguyen, T. D. Vuong, T. Van Toai, J. D. Lee, X. Wu, M. A. Rouf Mian, A. E. Dorrance, J. G. Shannon and H. T. Nguyen, "Mapping of Quantitative Trait Loci Associated with Resistance to Phytophthora sojae and Flooding Tolerance in Soybean," Crop Science, Vol. 52, No. 6, 2012, pp. 2481-2493. doi:10.2135/cropsci2011.09.0466

[33] F. Liu, C. R. Jensen and M. N. Andersen, "Hydraulic and chemical signals in control of leaf expansion and stomatal conductance in soybean exposed to drought stress," Functional Plant Biology, Vol. 30, No. 1, 2003, pp. 65-73. doi:10.1071/FP02170

[34] J. E. Specht, D. J. Hume and S. V. Kumindi, "Soybean Yield Potential: A Genetic and Physiological Perspective,” Crop Science, Vol. 39, No. 6, 1999, pp. 1560-1570. doi:10.2135/cropsci1999.3961560x

[35] M. N. Hunter, P. L. M. De Jabrun and D. E. Byth, "Response of Nine Soybean Lines to Soil Moisture Conditions Close to Saturation," Australian Journal of Experimental Agriculture and Animal Husbandry, Vol. 20, No. 104, 1980, pp. 339-345. doi:10.1071/EA9800339

[36] H. Banba, Y. Tanimura and I. Matsukawa, "A Report of Damage Due to Heavy Rainfall in Pulses in 1981," Report of the Hokkaido Branch, the Crop Science Society of Japan and Hokkaido Branch, the Japanese Society of Breeding, Vol. 22, No. 1, 1982, p. 55. (In Japanese) 